\title{
The Silicon Tracker of the LHCb experiment
}

\author{
B.Adeva ${ }^{5}$, M.Agari ${ }^{1}$, C.Bauer ${ }^{1}$, D.Baumeister ${ }^{1}$, A.Bay ${ }^{3}$, R.P.Bernhard ${ }^{4}$, R.Bernet ${ }^{4}$, J.Blouw ${ }^{1}$, B.Carron ${ }^{3}$, \\ Y.Ermoline $^{4}$, D.Esperante ${ }^{5}$, R.Frei ${ }^{3}$, J.Gassner ${ }^{4}$, W.Hofmann ${ }^{1}$, S.Jimenez-Otero ${ }^{3}$, K.T.Knöpfle ${ }^{1}$, $\underline{\text { S.Köstner }}^{4}$, \\ F.Lehner ${ }^{4}$, S.Löchner ${ }^{1}$, C.Lois ${ }^{5}$, M.Needham ${ }^{4}$, A.Perron ${ }^{3}$, V.Pugatch ${ }^{2}$, M.Schmelling ${ }^{1}$, B.Schwingenheuer ${ }^{1}$, \\ M.Siegler ${ }^{4}$, O.Steinkamp ${ }^{4}$, U.Straumann ${ }^{4}$, M.T.Tran ${ }^{3}$, P.Vazques ${ }^{5}$, A.Vollhardt ${ }^{4}$, D.Volyanskyy ${ }^{4}$, H.Voss $^{3}$
}

\begin{abstract}
LHCb is one of the experiments of the Large Hadron Collider at CERN, dedicated to B-physics and CP-violation measurements. To fully exploit the physics potential, a good tracking performance with high efficiency in a high particle density environment close to the beam pipe is required. Silicon strip detectors with large read-out pitch and long strips will be used for the LHCb Inner Tracker after the magnet and the Trigger Tracker station in front of the magnet. We report here about the design of the Silicon Tracker, test beam results and the electrical tests foreseen during module production.
\end{abstract}

Index Terms - LHCb, silicon, tracker, detector

\section{INTRODUCTION}

T HE LHCb detector [1], shown in Fig.1, currently under construction at the Large Hadron Collider at CERN, is designed to study the physics of b-flavoured hadrons. Precision measurements on CP-violating processes and rare decays with B-mesons play an important role in tests of the Standard Model and in searches for new physics beyond. The experiment will operate at a luminosity of $2 \cdot 10^{32} \mathrm{~cm}^{-2} \mathrm{~s}^{-1}$ with an expected production of $10^{12} \mathrm{~b} \bar{b}$-pairs per year.

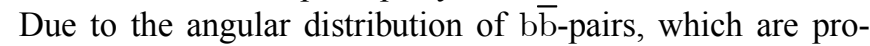
duced predominantly at low polar angles, $\mathrm{LHCb}$ is designed as a single arm forward spectrometer with an acceptance of $15 \mathrm{mrad}$ to $300 \mathrm{mrad}$ in the bending plane. A silicon-microstrip vertex detector [3] surrounding the interaction region and the Trigger Tracker (TT-station) [1] located in the fringe field in front of the $4 \mathrm{Tm}$ dipole magnet together with the tracking stations downstream the magnet are used to reconstruct charged particle trajectories. To provide a higher granularity in the high particle density region near the beam-pipe, the tracking stations T1-T3 are split into the Inner Tracker [2], applying siliconmicro-strip technology, surrounded by the Outer Tracker [4] built from straw-tubes. Although the Inner Tracker covers just $1.3 \%$ of the total area it will contain almost $20 \%$ of the tracks to be reconstructed. The occupancy is expected to be less than $2 \%$. The TT-station covers the full acceptance of the

\footnotetext{
${ }^{1}$ MPI for Nuclear Physics, Saupfercheckweg 1,69117 Heidelberg, Germany

${ }^{2}$ Ukranian Academy of Science, UA-252 143, Kiev 143, Ukraine

${ }^{3}$ LPHE, EPFL Lausanne, Bât. des Sciences Physiques, 1015 Dorigny, Switzerland

${ }^{4}$ Physik-Institut der Universität Zürich, Winterthurerstr. 190, 8057 Zürich, Switzerland

${ }^{5}$ Universidad de Santiago de Compostela, Campus Universitario Sur, 15706 Santiago de Compostela, Spain
}

Corresponding author: Stefan.Koestner@cern.ch

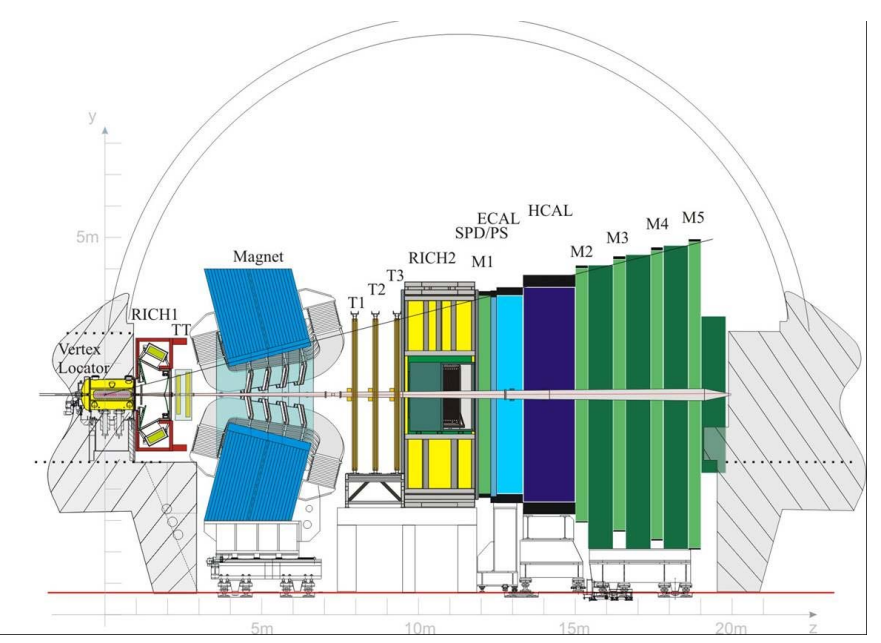

Fig. 1. Schematic view of the LHCb experiment. The TT-station is located in front of the dipole magnet. The Inner Tracker is part of the three tracking stations downstream the magnet, surrounding the beam pipe.

experiment and is used to measure the transverse momentum of large impact parameter tracks in the second trigger level. The operation temperatures of the TT-station and the Inner Tracker are foreseen to be $5^{\circ} \mathrm{C}$ in order to reduce the noise contribution due to leakage currents caused by irradiation. Fluences of up to $5.5 \times 10^{13} \mathrm{~cm}^{-2}$ of $1 \mathrm{MeV}$ neutron equivalent are expected at the centre of the TT-station in 10 years of operation.

The Silicon Tracker project comprises the Inner Tracker and the Trigger Tracker. The LHCb detector is completed by two RICH detectors for particle identification, electro-magnetic and hadronic calorimeters and muon detectors.

Simulation studies have shown that the momentum resolution in $\mathrm{LHCb}$ is dominated by multiple scattering over a wide range of momenta. This results in a spatial resolution requirement which is met by silicon micro-strip detectors with a read-out pitch of the order of $200 \mu \mathrm{m}$. Large read-out pitch and long read-out strips, adapted to the expected hit occupancies, are used throughout the Silicon Tracker in order to reduce the number of read-out channels and hence the costs. The Silicon Tracker covers a total area of $12.2 \mathrm{~m}^{2}$ with $270 \mathrm{k}$ read-out channels.

\section{INNER TRACKER DESIGN}

Each Inner Tracker station [2] consists of four individual detector boxes which are arranged around the beam pipe as 


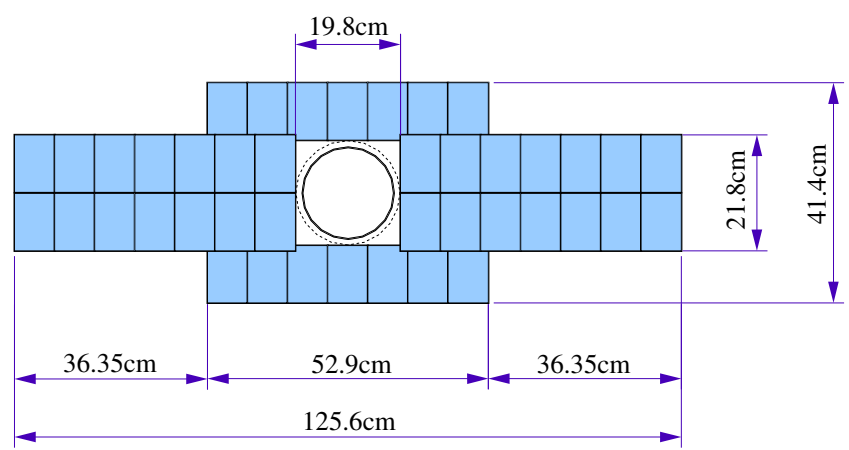

Fig. 2. The layout of one Inner Tracker station consisting of four individual detector boxes.

shown in Fig.2. The side boxes contain Si-ladders that are $22 \mathrm{~cm}$ long and are built out of two sensors. The detector boxes above and below the beam-pipe employ single-sensor ladders. The geometry provides sufficient granularity for the high particledensity region around the beampipe. Each detector box houses 28 Si-ladders in four detection layers, with the two inner layers placed with a $\pm 5^{\circ}$ stereo angle. The boxes provide electrical and thermal insulation.

Single-sided AC coupled $\mathrm{p}^{+} \mathrm{n}$ silicon strip sensors are used, which are $7.8 \mathrm{~cm}$ wide and $11 \mathrm{~cm}$ long. The sensor thickness is chosen to be $320 \mu \mathrm{m}$ for the short ladders and $410 \mu \mathrm{m}$ for the two sensor ladders in order to ensure sufficient signal in the presence of the increased noise due to increased load capacitance. The read-out pitch is $198 \mu \mathrm{m}$ and each ladder is read out via 3 Beetle [6] read-out chips. The Beetle is a 128 channel custom made analog read-out chip using commercial $0.25 \mu \mathrm{m}$ CMOS technology with a radiation hard design. It operates at $40 \mathrm{MHz}$ and saves events into a pipeline with a maximum latency of 160 clock cycles. The shaping time can be varied via an internal programmable register $\left(V_{f s}\right)$ which changes the feedback resistance. The analog data of triggered events are sent via a $5 \mathrm{~m}$ long copper cable to service boxes located just outside of the acceptance of the experiment, where the signals are amplified and digitized before they are multiplexed onto a $100 \mathrm{~m}$ digital optical link.

\section{TRIGgER TRACKER DESIGN}

The TT-station consists of four layers arranged in two half stations, TTa and TTb, separated by $30 \mathrm{~cm}$ along the beam axis and enclosed in a single box providing electrical and thermal shielding.

The box is split in order to retract the two halves to allow access to the beam-pipe for the bake-out procedure. As in the Inner Tracker, the first and the fourth layer have vertical readout strips, while the inner two layers have a stereo angle of $\pm 5^{\circ}$.

For the sensors used for the TT-station, the most cost efficient solution which fully matches our requirements, is to employ a sensor design from the outer barrel of the CMS silicon tracker [7]. The $500 \mu \mathrm{m}$ thick sensors have a width of $9.6 \mathrm{~cm}$

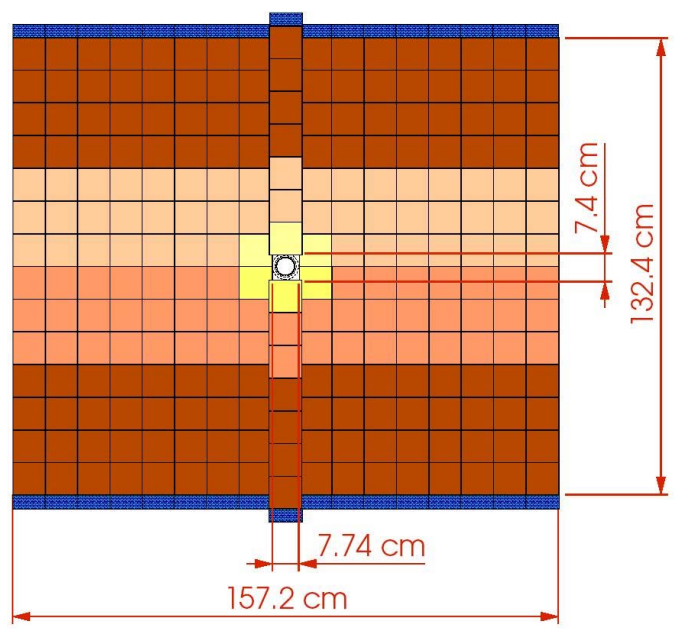

Fig. 3. The layout of one layer in the TT-station (TTb) is shown. The entire $\mathrm{LHCb}$ angular coverage is provided by silicon strip detectors. Short read-out sectors are used in the centre around the beam pipe, longer read-out sectors of up to four sensors elsewhere.

and a length of $9.4 \mathrm{~cm}$. The 512 read-out strips with a pitch of $183 \mu \mathrm{m}$ are read out via 4 Beetle [6] read-out chips.

The sensors are arranged in read-out sectors of one- and two sensors in the centre around the beam pipe as indicated in Fig.3. Read-out sectors consisting of three sensors bonded together are used near the centre of the bending plane and four sensors, i.e. $38 \mathrm{~cm}$ long strips, in the outer area of the detector. The read-out hybrids are located at the outer edge of the detector, outside of the acceptance. The inner sectors are connected to the read-out hybrids via $40 \mathrm{~cm}$ long Kapton interconnect cables. A cable of $58 \mathrm{~cm}$ length connects to the single sensor near the beampipe. This results in a load capacitance of up to $57 \mathrm{pF}$ for the pre-amplifier.

\section{Test Beam Results}

Several prototype modules with different sensor thickness, strip length and implant over pitch ratio were built and tested in a $120 \mathrm{GeV} \pi^{-}$beam at CERN, in order to study the resulting noise behaviour w.r.t. the input load capacitance, the overall $\mathrm{S} / \mathrm{N}$ ratio, the charge loss in the inter-strip region for the large pitch detectors and the shaping time. These results were used to extrapolate the performance to the actual detector layout.

Starting with the expected charge generated in the sensors, the equivalent noise charge for different strip capacitances is deduced from the observed $\mathrm{S} / \mathrm{N}$ values. The results are shown in Fig. 4 together with the expectation from laboratory measurements, where load capacitances have been connected to the input of the Beetle chip on a test-bench. Good agreement is found for the dependence of the noise on the load capacitance with a small offset indicating an additional constant noise source in the test-beam setup. This dependence can be used to extrapolate the $\mathrm{S} / \mathrm{N}$ expectation for modules with increased load capacitances due to Kapton interconnect cables or additional sensors. 


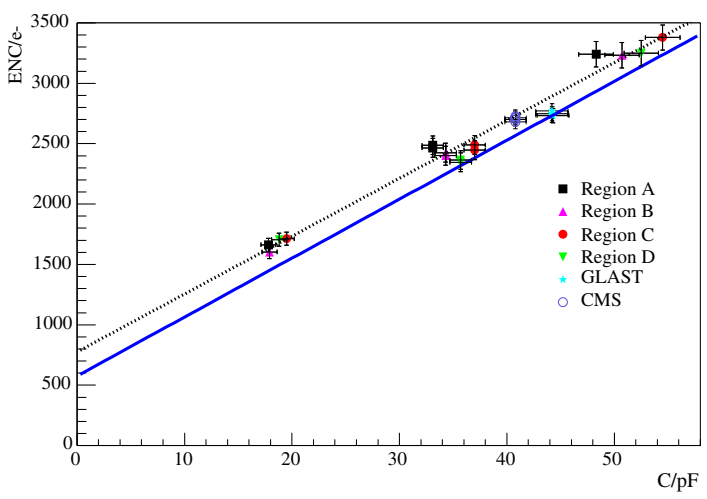

Fig. 4. The equivalent noise charge obtained with the Beetle read-out chip depending on its input load capacitance. Regions A-D correspond to different implant and pitch widths on LHCb Inner Tracker prototype sensors. Other measurements were taken with modules built from CMS [7] and GLAST [8]. The dashed line is the best fit to the test-beam data while the solid line is the expectation from laboratory measurements.

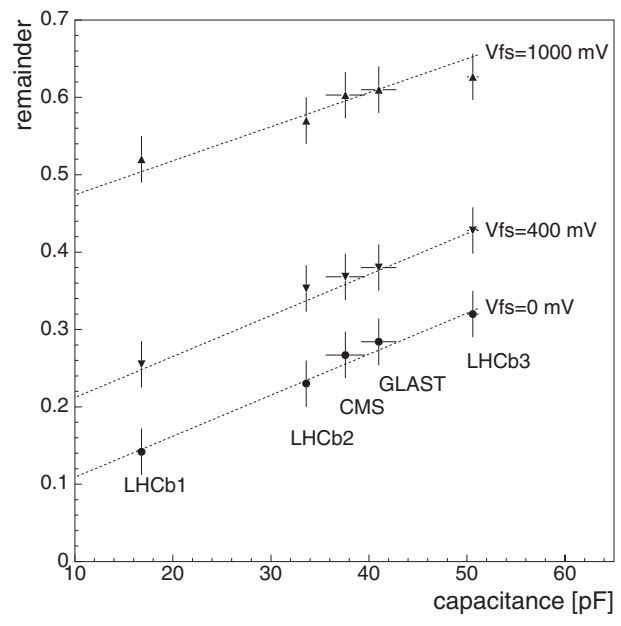

Fig. 5. Signal remainder after $25 \mathrm{~ns}$, i.e. one bunch crossing.

The shaping time was varied by means of the $V_{f s}$ setting in order to study the remaining signal $25 \mathrm{~ns}$ after the maximum, i.e. in the consecutive bunch crossing. The signal width increases with increasing load capacitance as seen in Fig.5. It is found that with $V_{f s}$ settings below $400 \mathrm{mV}$ the signal remainders can be kept below $30 \%$ and $50 \%$ as required for the Inner Tracker and the TT-station, respectively.

Fig. 6 shows measurements of the $\mathrm{S} / \mathrm{N}$ ratio for different relative positions of the incident particle w.r.t. the read-out strips for a module built out of three CMS-OB2 sensors. The decrease of the $\mathrm{S} / \mathrm{N}$ ratio in between the strips is due to a charge loss that was already observed in previous measurements [2]. The final detector design requires the $\mathrm{S} / \mathrm{N}$ ratio to be high enough also in between two read-out strips. To obtain full cluster finding efficiency at a reasonable noise-rate, a $\mathrm{S} / \mathrm{N}$ ratio of 10 is required. Adding a safety margin, the $\mathrm{S} / \mathrm{N}$ value should be higher than 12 over the whole detector.

In a recent test-beam period, a TT-module consisting of

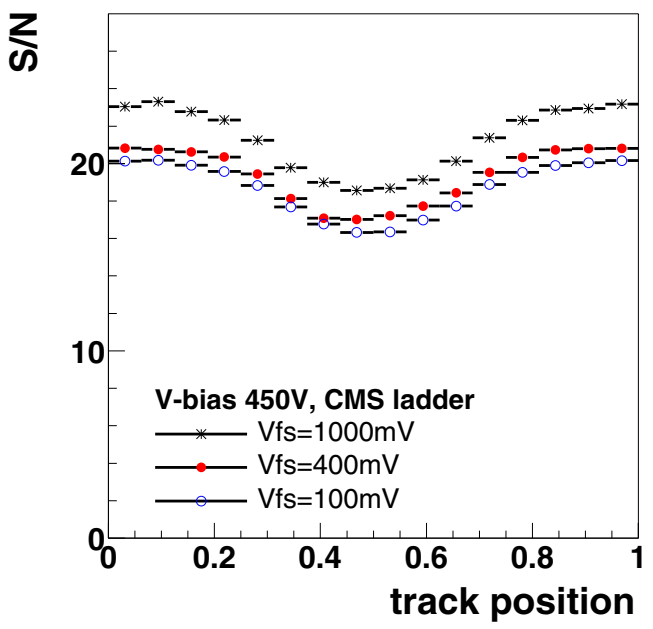

Fig. 6. The $\mathrm{S} / \mathrm{N}$ for different shaping times, depending on the relative positions of the incident particle w.r.t. the read-out strips. Positions 0 and 1 correspond to the center of the read-out strip.

three CMS-OB2 sensors and a $40 \mathrm{~cm}$ Kapton interconnect cable has been tested. Preliminary results indicate the signal-to-noise behaviour is as expected. The performance of irradiated ladders was also studied in this test-beam. The irradiation dose for the Inner Tracker after 10 years is expected to be around $1 \mathrm{Mrad}$ or $9 \cdot 10^{12} \mathrm{~cm}^{-2}$ of $1 \mathrm{MeV}$ neutron equivalent. For a $\mathrm{LHCb}$ Inner Tracker prototype, irradiated up to this dose, full charge collection efficiency was obtained at a bias voltage of around 350 Volts. Preliminary results show no significant charge loss in the irradiated sensor, though an increase in noise.

\section{Electrical Tests for Production}

Pre-production of the Silicon Tracker will start soon. An efficient detection scheme of potential defects of the ladders, namely shorted channels, broken bonds and pinholes, is crucial for the quality. In order to study possible detection methods, defects were introduced in a prototype module consisting of three $410 \mu \mathrm{m}$ sensors. The DC-pad and AC-pad of one channel were bonded together in order to simulate punch throughs in the dielectric of the microstrips. Wire bonds were removed on different locations of the ladder and AC-pads of neighbouring channels bonded together.

These defects cause a change in the load capacitance and thus affect the response of the Beetle read-out chip. Pulseshape scans were performed using the internally generated test pulses of the Beetle. Defects were identified as channels whose pulse height lies more than three standard deviations outside of the general distribution of the other channels. As shown in Fig.7, a linear fit was used to allow for an overall linear trend over the read-out channels. Channels with broken bonds have less capacitance which increases the gain in the amplifier. Shorted channels appear to have a lower peak height, due to the increase of the capacitance, which makes it easy to distinguish between the two defects. Additional information can be gained from the width of the pulse shape. The distribution of the ratio of the 


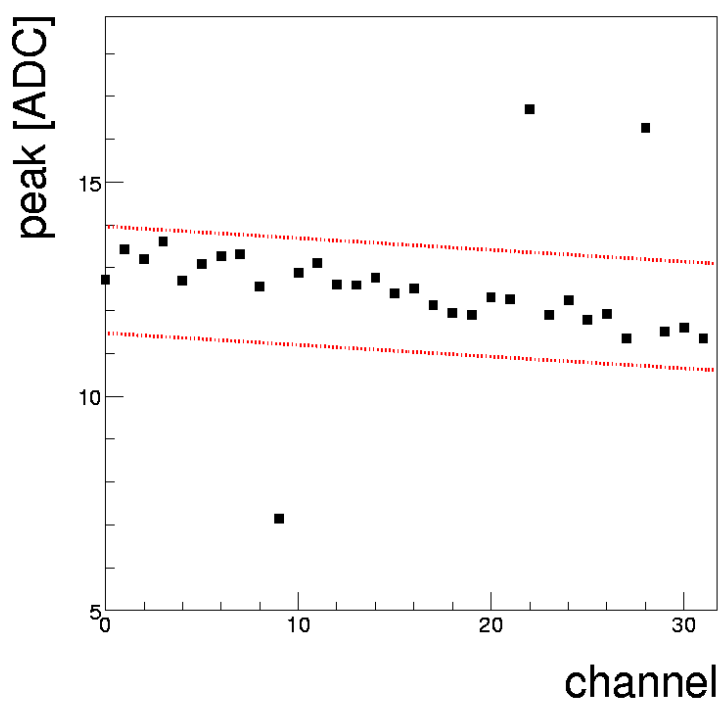

Fig. 7. Distribution of the peak height of the internally generated test pulse. For two channels with a broken bond between middle and first sensor the pulse height is higher than the average. For a shorted channel the pulse height is smaller due to the increase of load capacitance. The dashed lines indicate three standard deviations to a linear fit after discarding the defects. Every fourth channel is plotted due to the four-fold testpulse pattern of the Beetle 1.2 chip.

signal remainder $25 \mathrm{~ns}$ after the peaking time to the peak height is shown in Fig.8.

For minimizing statistical fluctuations in the distribution of the various parameters of the pulse shape, a fitting function for the pulse shape in a range as wide as possible had to be found. A simplistic model of the Beetle as a non-ideal pre-amplifier convoluted with a CR-RC shaper has shown much better results than fitting a gaussian in a small range around the peak.

While for depleted sensors, the artificially introduced pinholes have shown no significant noise pattern nor any deviations in the pulse shapes, the effect of the pinholes could be seen after applying a small forward bias of -0.5 Volts. To prevent from potential damage the current source was limited. In this case the response of the Beetle chip to test pulses for channels with pinholes was almost zero.

Compared to noise plots, pulse shapes from internal test pulses are more significant and conclusive w.r.t the type of defect. However, a final detection procedure and definition of cuts will be given after more experience is gained during the pre-production. Complementary tests, e.g. laser teststand and wire pick-up tests will be performed.

\section{CONCLUSION}

The LHCb Silicon Tracker is designed using silicon strip detectors with up to $38 \mathrm{~cm}$ long read-out strips, a large pitch of $\approx 200 \mu \mathrm{m}$ and fast read-out electronics adapted to the $40 \mathrm{MHz}$ bunch crossing rate at the LHC. Test-beam measurements of the prototype modules have shown performance according to expectations and within the specification of the $\mathrm{LHCb}$ experiment. The internal test pulse generator of the Beetle pre-amplifier chip can be utilized for a stand-alone procedure to detect defects

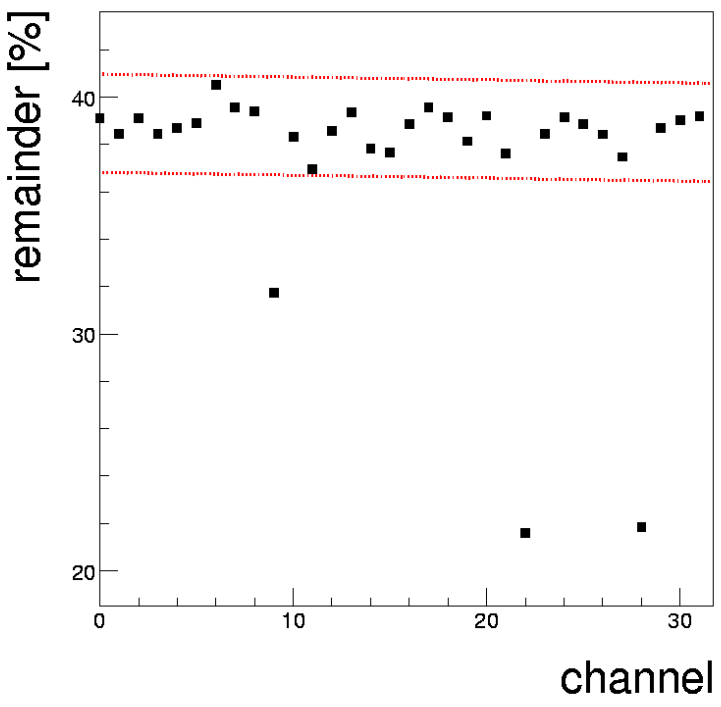

Fig. 8. Distribution of the signal remainder, $25 \mathrm{~ns}$ after the peaking time. Shorts and broken bonds appear as having a smaller remainder than the average. The dashed lines indicate three standard deviations to a linear fit after discarding the defects. Due to the particular testpulse pattern every fourth channel is plotted.

during the production. It could be shown that shorts, broken wire bonds and pinholes can be detected at high significance.

\section{ACKNOWLEDGMENT}

We would like to thank Pawel Jalocha for providing the hardware and support during the electrical characterization.

\section{REFERENCES}

[1] LHCb Collaboration: LHCb Reoptimized Detector, Design and Performance, CERN/LHCC 2003-030.

[2] LHCb Collaboration: LHCb Inner Tracker, CERN/LHCC 2002-029.

[3] LHCb Collaboration: $L H C b$ VELO, CERN/LHCC 2001-011.

[4] LHCb Collaboration: LHCb Outer Tracker, CERN/LHCC 2001-024.

[5] M. Agari et al: Testbeam measurements on prototype ladders for the $\mathrm{LHCb}$ TT station and Inner Tracker, LHCb note 2003-082;

J. Gassner, M. Needham, O. Steinkamp: Layout and Performance of the LHCb TT station, LHCb note 2003-140.

[6] U. Trunk et al., CERN/LHCC 2003-055.

[7] J.-L. Agram et al.: The Silicon Sensors for the Compact Muon Solinoid Tracker - Design and Qualification Prodedure, CMS note 2003-015.

[8] GLAST Collaboration,

http://scipp.ucsc.edu/groups/glast/detector. 